\title{
A Possible Coronavirus Isolation from Multiple Sclerosis Autopsy Material
}

\author{
J. S. BurKS ${ }^{1}$, B. DeVAld ${ }^{1}$, L. JANKovsky ${ }^{2}$, and J. Gerdes ${ }^{2}$
}

\section{Introduction}

While epidemiologic and serologic evidence suggests a viral etiology of multiple sclerosis (MS), virus isolations have yet to be confirmed. Our laboratory has possibly isolated a coronavirus from two MS patients. Virus was isolated from two of nine MS patients' brains at autopsy. This paper reports the isolation methods, the attempts to identify the virus as a coronavirus, and a preliminary serologic survey investigating the presence of antibody to this virus in MS and control populations.

\section{Case Report and Isolate No. 1}

The patient was a 55-year-old woman with a 28-year history of MS. Her most recent MS symptoms were related to brain stem dysfunction. An autopsy performed within $4 \mathrm{~h}$ following death revealed typical MS plaques in the cerebrum, cerebellum, brain stem, and spinal cord. Histologic sections of medulla and pons showed areas of demyelination surrounded by reactive astrocytes.

Fresh, unfrozen brain stem from autopsy material was prepared as a sterile $10 \%$ homogenate and inoculated intracerebrally (IC) $(0.03 \mathrm{ml})$ into ten weanling BALB/c mice. Seven of the ten mice died between 2 and 6 months after inoculation. Some moribund mice exhibited seizures, myoclonic jerks, and limb paralysis. When fresh brain stem material from a mouse dying 99 days after inoculation was inoculated IC into another group of weanling $\mathrm{BALB} / \mathrm{c}$ mice, the incubation period was decreased to approximately 50 days. Mouse brain stem material obtained from these mice was inoculated into newborn BALB/c mice IC with resultant disease and death in 12 days. Subsequently, on serial passages, the incubation period decreased to 3-5 days. The freezing of infected weanling and newborn mouse brain material during early passage attempts usually resulted in a loss of transmissibility, whereas fresh, unfrozen mouse brain material consistently produced illness.

Histologic studies in the few moribund adult mice have been negative. However, in newborn BALB/c mice, the agent induced multifocal areas of spongy change with relative sparing of neurons and axons. After the fourth passage of this isolate in newborn mice, necrotizing thymic, hepatic, and enteric lesions were noted. These lesions were not present in earlier passages.

1 Veterans Administration Hospital, 1055 Clermont Street, Denver, Colorado 80262/USA

2 University of Colorado Medical Center, Denver, Colorado 80262/USA 


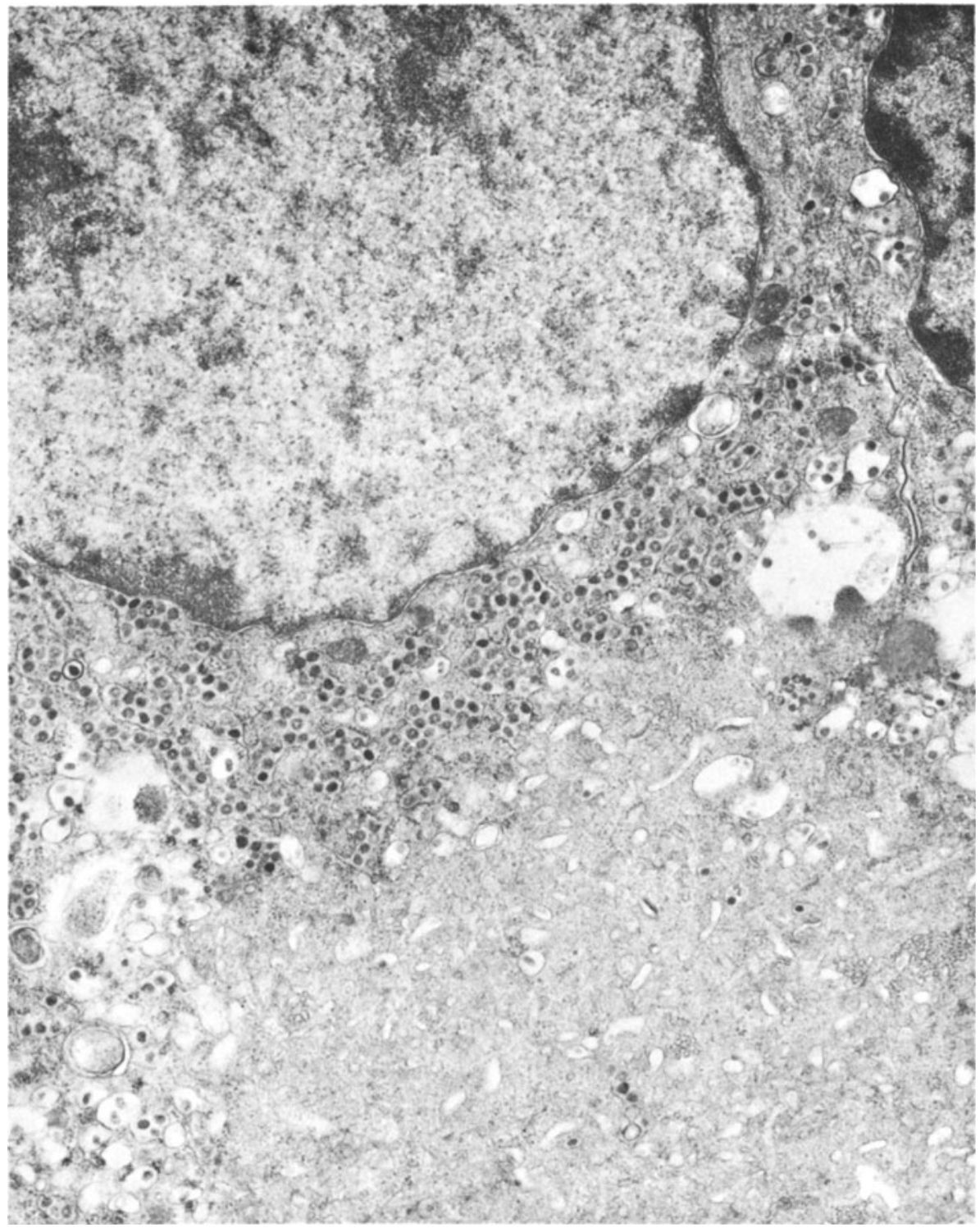

Fig. 1. Electron microscopy of a giant cell with Isolate I reveals $80-120 \mathrm{~nm}$ diameter viral particles in vacuoles and among profiles of the smooth endoplasmic reticulum. $\mathrm{x} 28,375$

Electron-microscopic examination of livers from infected suckling mice has revealed numerous $80-120 \mathrm{~nm}$ virus-like particles within vacuoles. Rarely, brains from the same infected suckling mice revealed similar structures in spongy areas.

Serologic studies on IC-inoculated weanling mice showed no consistent antibody response to pneumonia virus of mice, Sendai virus, reovirus type 3 , mouse hepa- 


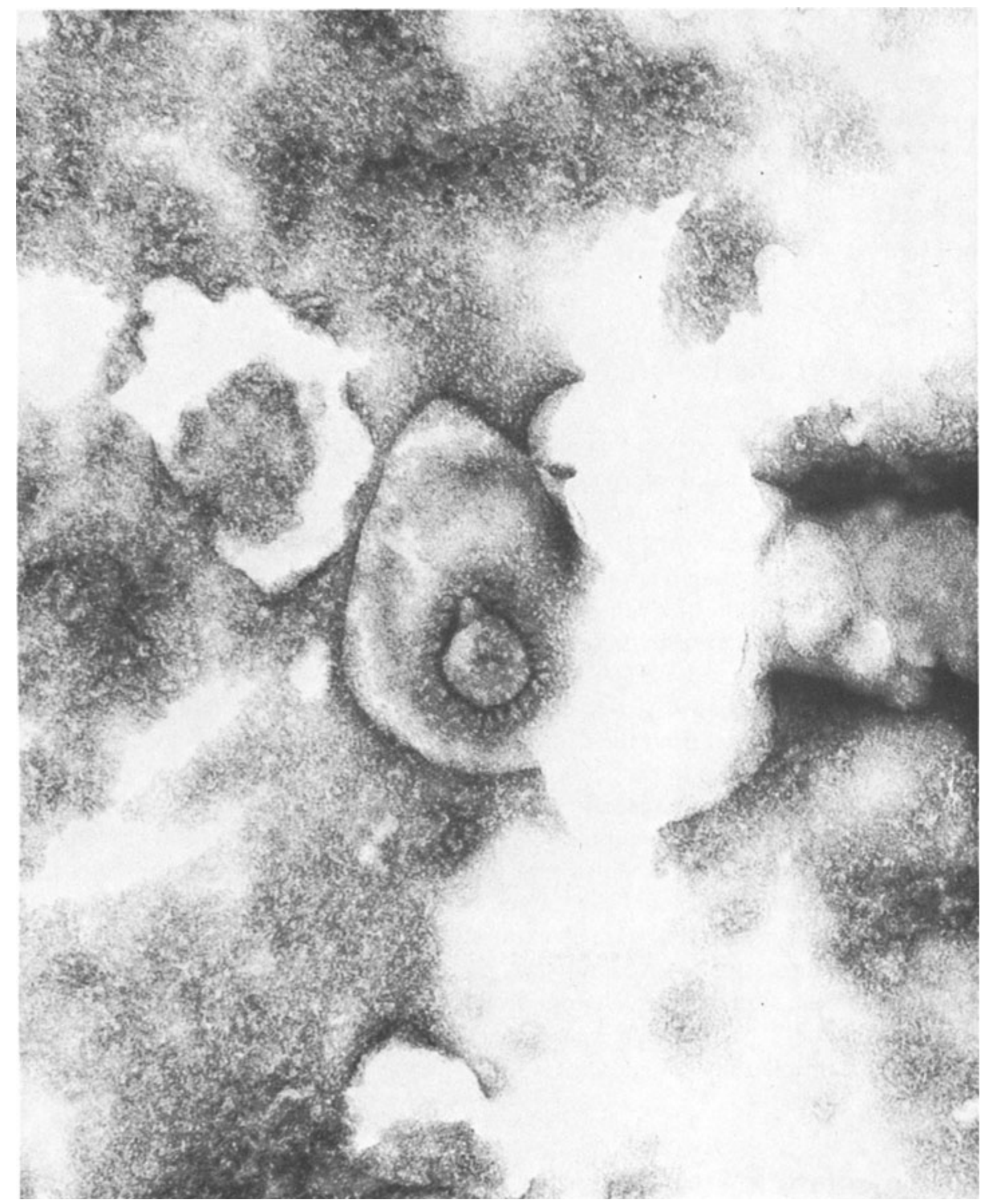

Fig. 2. Negative stain electron microscopy of virus Isolate I reveals the pleomorphic 80-100 $\mathrm{nm}$ core surrounded by approximately $10 \mathrm{~nm}$ club-shaped spikes. $\mathrm{x} 180,000$

titis virus, mouse encephalomyelitis virus, $\mathrm{K}$ virus, polyoma, ectromelia, minute virus of mice, adenovirus, or lymphocytic choriomeningitis virus when assayed by Microbiological Associates Laboratory, Bethesda, Maryland, USA. No cytopathic effect was noted when infected brain material homogenate was inoculated into tissue cultures of Rhesus monkey kidney, African green monkey kidney, human embryonic lung, NCTC-1469, mouse L cells, primary bovine embryonic kidney, primary 
rabbit kidney, W138, BSC-1, MDCK (canine kidney), HELA, HEP-2, VERO, rabbit kidney, and primary human amnion.

When infected suckling mouse brain homogenates were inoculated into $17 \mathrm{CL}-1$ cells (a spontaneously transformed 3T3 cell line), syncytial formation (giant cells) was observed. Electron microscopy on these cultures revealed viral particles similar to those seen in infected suckling mouse liver and brain (Fig. 1). Negative stain electron microscopy on infected $17 \mathrm{CL}-1$ cells revealed virus particles resembling coronavirus (Fig. 2).

\section{Case Report and Isolate No. 2}

The patient was an 89-year-old woman with a 40 to 50 year history of neurological problems involving mainly corticospinal tracts with resultant weakness. Also, she demonstrated cerebellar dysfunction and extraocular movement paresis. Her course was slowly progressive for the last 30 years without exacerbations or remissions. An autopsy performed within $4 \mathrm{~h}$ following death revealed bilateral pneumonia and demyelinated areas in the periventricular white matter, brain stem, cerebellum, and spinal cord. A mild glial reaction was noted around most demyelinated areas.

Fresh, unfrozen autopsy material was prepared as a sterile $10 \%$ homogenate and inoculated into weanling BALB/c mice IC and $17 \mathrm{CL}-1$ cells as described in case report 1 . Fresh material from the frontal lobe, occipital lobe, parietal lobe, cerebellum, midbrain, pons, cervical spinal cord, thoracic spinal cord, lumbosacral spinal cord, and lymph nodes was used in both mice and $17 \mathrm{CL}-1$ experiments. All mice remained healthy but on subculture No. 12, synctial formation was noted in the 17 CL-1 cells inoculated with deep frontal lobe material. Coronavirus-like particles, similar to those seen as Isolate I were noted by electron microscopy in the tissue culture cells. However, negative stain electron microscopy of tissue culture supernatant has not been successful in revealing virus particles, probably due to low virus concentration in the supernatant. The other eight central nervous system (CNS) areas from this patient's brain which were inoculated into mice and $17 \mathrm{CL}-1$ cells produced no viral isolation.

\section{Other Isolation Attempts}

Although no littermate controls were inoculated with fresh, unfrozen non-MS autopsy material at the time of the initial transmission studies, numerous other mice within the colony have been inoculated with human brain material. This includes two MS patients whose brain material was frozen before inoculation into mice. Since the first virus isolation, we have inoculated 97 areas of CNS tissue from five additional MS patients into weanling mice and 17 CL-1 cells. No virus has yet been detected in this material. Also, we have inoculated fresh, unfrozen material from 28 areas of the CNS from 12 non-MS patients into mice and $17 \mathrm{CL}-1$ cells with negative results. 


\section{Serologic Studies}

A plaque neutralization assay was developed using the second virus isolate to evaluate the serum and spinal fluid antibody concentrations to this virus in MS patients and a control population. Serum was diluted $1: 20$ and spinal fluid was diluted $1: 2$ for these tests. Greater than $50 \%$ plaque reduction was considered as evidence for the presence of antibody to the virus. Spinal fluid from five of 140 (3.5\%) MS patients (including the two virus-positive patients) had antibody to the second virus isolate. No spinal fluid from 43 control patients neutralized the virus. Serum from 85 of $102(83 \%)$ MS patients neutralized the virus at a $1: 20$ dilution, whereas only ten out of 55 (18\%) serums from control patients neutralized the virus (Table 1).

Table 1. Neutralization of virus isolate II by serum and spinal fluid from MS and control patients

\begin{tabular}{lll}
\hline & Serum $^{\text {a }}$ & CSF $^{\mathrm{b}}$ \\
\hline MS patients & $85 / 102$ & $5 / 140$ \\
& $(81 \%)$ & $(3.5 \%)$ \\
Control patients & $10 / 55$ & $0 / 43$ \\
& $(18 \%)$ & $(0 \%)$ \\
\hline
\end{tabular}

a Serum: Number of serums (1:20 dilution) demonstrating $>50 \%$ plaque reduction against Isolate No. 2 / the total number of serum samples tested

b $\mathrm{CSF}=$ Number of cerebrospinal samples ( $1: 2$ dilution) demonstrating $>50 \%$ plaque reduction against Isolate No. $2 /$ the total number of CSF samples tested

\section{Discussion}

The isolation of coronavirus-like agents using different techniques and two MS patients presents several unanswered questions. First, what were the origins of the isolate? Second, if the isolates did originate from the patients, what is their relationship to the pathogenesis of multiple sclerosis? Lastly, why should these isolation attempts have been successful when previous attempts have failed?

There are several possible explanations for the origin of these isolates. First, they might be contaminating agents from sources other than patient brain material. Endogenous mouse coronaviruses are notorious for contaminating isolation and transmission studies. The second isolate is unlikely to be a mouse contaminant virus, since coronavirus particles have not been found in $17 \mathrm{CL}-1$ cells. Despite the precautions taken, the isolates might represent laboratory contaminants.

The possibility that the isolates are coronaviruses from the MS patients is supported by several facts. First, the presence of neutralizing antibody to this virus in 
the spinal fluid of the two virus-positive MS patients indicates possible viral specific antibody production within the CNS of these patients. Second, a statistically significant, higher percent of MS patients shows antibody to this virus in the serum when compared to control patients' serum. Third, coronaviruses are an attractive candidate virus for MS, since mouse hepatitis virus, another coronavirus, causes a demyelinating and remyelinating disease when inoculated IC into mice [2]. Finally, coronavirus particles have been previously identified by electron microscopy in brain tissue of one MS patient previously [1].

If the virus originated from the MS patients' brains, its relationship to the pathogenesis of multiple sclerosis would still remain questionable. Activation of a coronavirus may have been related to a terminal event or to a latent infection which is unrelated to multiple sclerosis. Additional isolations from MS patients in other laboratories are essential before an association can be made between a coronavirus and MS.

The success of our virus isolations may be related to the marked sensitivity of the virus to freezing, since the virus was not isolated when brain material was frozen. Many viruses lose some degree of infectivity after freezing. However, most viruses are numerous enough so that a loss of $90 \%$ of the infected particles would not hamper isolation attempts. However, if only small numbers of infectious virus particles are present, freezing the material may decrease the number of particles enough to prohibit isolation. Therefore, the use of fresh brain material is a possible methodological advance which may increase the chances of the recovery of a virus(es) in MS.

Emphasis should be placed on the relative rarity of our isolations. To date we have inoculated 99 fresh CNS homogenates from seven MS patients into mice or tissue culture cells and have isolated the virus in only two of these attempts. We have found antibody to the virus in only five of 140 spinal fluids from MS patients. If coronaviruses play a role in MS, an explanation for our lack of repeated success in isolations may be that the virus resides in a latent or defective state in the CNS.

While we do not claim to have isolated an agent related to MS, we do hope that this report will stimulate other investigators to use fresh unfrozen brain material from multiple CNS sites in future isolation attempts in MS. Coronaviruses should be looked for specifically in future isolation attempts from other laboratories.

Acknowledgments. This research was supported by a grant from the Kroc Foundation of California and by Veteran's Administration Grant No. 1169.

\section{References}

1. Tanaka R, Iwasaki Y, Koprowski, HJ (1976) Intracisternal virus-like particles in brain of a multiple sclerosis patient. J Neurol Sci 28:121

2. Weiner LP (1973) Pathogenesis of demyelination induced by a mouse hepatitis virus (JHM virus). Arch Virol 28:298 